\title{
In vitro effects of levosimendan on muscle of malignant hyperthermia susceptible and non-susceptible swine
}

\author{
Frank Schuster ${ }^{*} \mathbb{D}$, Stephan Johannsen, Susanne Isbary, Ismail Türkmeneli and Norbert Roewer
}

\begin{abstract}
Background: The calcium sensitizer levosimendan is increasingly used to improve hemodynamics in patients with acutely decompensated heart failure. By binding to cardiac troponin C the conformation of the calcium-troponin C complex is stabilized, which leads to acceleration of actin-myosin crossbrigde formation and increased force generating capacity of muscle fibers. Besides indications in cardiac failure, beneficial effects of levosimendan in skeletal muscle disorders are currently evaluated. The aim of this study was to investigate differential effects of levosimendan on skeletal muscle of pigs with and without susceptibility to malignant hyperthermia (MH) in order to identify possible risks of this emerging drug for patients with predisposition to $\mathrm{MH}$.
\end{abstract}

Methods: Muscle bundles of 17 pigs (9 MH susceptible (MHS); $8 \mathrm{MH}$ non-susceptible (MHN)) were excised under general anesthesia and examined in the tissue bath with increasing concentrations of levosimendan $(0.065 ; 0.125 ; 0.5$; 1.0; 10 and $50 \mathrm{\mu g} / \mathrm{ml}$ ). Baseline tension and twitch force were monitored continuously. Data are presented as median and interquartile range. Statistical evaluation was performed using D'Agostino \& Pearson test for normal distribution and student's $t$ test and 2-way ANOVA for differences between the groups. $P<0.05$ was considered significant.

Results: There were no differences between the groups concerning length, weight, initial twitch force and pre-drug resting tension of the investigated muscle strips. After an initial decrease in both groups, twitch amplitude was significantly higher in MHN (- 3.0 [-5.2-0.2] mN) compared to MHS $(-7.5[-10.8--4.5] \mathrm{mN})(p=0.0034)$ muscle at an applied levosimendan concentration of $50 \mathrm{\mu g} / \mathrm{ml}$. A marked increase in resting tension was detected following levosimendan incubation with $50 \mathrm{\mu g} / \mathrm{ml}$ in MHS muscle bundles (3.3 [0.9-6.1] mN) compared to MHN $(-0.7[-1.3-0.0] \mathrm{mN})(p<0.0001)$.

Conclusions: This in vitro investigation revealed the development of significant contractures in muscle bundles of MHS pigs after incubation with levosimendan. However, the effect appeared only at supra-therapeutic concentrations and further research is needed to determine the impact of levosimendan on MHS individuals in vivo.

Keywords: Malignant hyperthermia, Levosimendan, Muscle, Pigs, In vitro contracture test

\section{Background}

Levosimendan, a calcium sensitizer and adenosine triphosphate dependent potassium channel opener mainly used for treatment of acute heart failure, improves myocardial contractility and enhances the sensitivity of myofilaments to calcium. The increase of cardiac inotropy is mediated through the binding to slow skeletal and cardiac troponin $C[1,2]$, while associated vasodilatation and cardioprotective effects are based on the interaction

\footnotetext{
* Correspondence: schuster_f@ukw.de

Department of Anesthesia and Critical Care, University of Wuerzburg,

Oberduerrbacher Str. 6, D-97080 Wuerzburg, Germany
}

of potassium depended adenosine triphosphate channels with cardiac mitochondria and vascular smooth muscle [3]. Besides the positive inotropic modulation of cardiac function, there is growing evidence for beneficial effects of levosimendan on further organ systems. For instance, improvement of contractility of slow-twitch muscle fibers and neuromechanical efficiency of the diaphragm in animals and humans was observed following levosimendan application $[4,5]$. Furthermore, a positive inotropic effect in skeletal muscle diseases was detected in vitro for calcium sensitizers due to an increased calcium mediated muscular contraction [6]. On the contrary, a

(C) The Author(s). 2018 Open Access This article is distributed under the terms of the Creative Commons Attribution 4.0 International License (http://creativecommons.org/licenses/by/4.0/), which permits unrestricted use, distribution, and 
pre-existing imbalance of calcium homeostasis might be associated with severe side effects for the patient, if calcium-modulating or sensitizing drugs are used.

In animals and humans susceptible to malignant hyperthermia $(\mathrm{MH})$ exposure to triggering agents like volatile anesthetics and depolarizing muscle relaxants may lead to a life-threatening uncontrolled sarcoplasmic calcium release, resulting in hypoxemia, hypercapnia, tachycardia, muscular rigidity, lactic acidosis, hyperkalemia and hyperthermia. Mutations in the gene of the ryanodine receptor subtype 1 (RYR1) or of the dihydrophyridine receptor (CACNA1S) have been identified as genetic cause for the pathologic alteration of intracellular calcium handling [7]. Although the drug-induced deterioration of calcium homeostasis by calcium efflux from the sarcoplasmic reticulum represents the main pathomechanism of a developing $\mathrm{MH}$ crisis, different molecular pathways leading to an increase in intracellular calcium concentration may influence the metabolic state in $\mathrm{MH}$-susceptible (MHS) muscle cells. Hence, administration of calcium modulating or sensitizing drugs might result in an unpredictable muscular response in individuals susceptible to $\mathrm{MH}$.

In the present study, the authors investigated differential effects of the calcium sensitizer levosimendan on skeletal muscle bundles of MHS and MH-non-susceptible (MHN) pigs, hypothesizing that with clinically used levosimendan concentrations no significant muscular contractures would occur in MHS or MHN muscle bundles in vitro, excluding a possible risk of this emerging drug for MHS individuals.

\section{Methods}

\section{Experimental protocol}

With approval of the local animal care committee (Government of Unterfranken, Wuerzburg, Germany, No. 39/13), homozygous MHS and MHN Pietrain pigs were investigated. The pigs were purchased from a local farmer (Farm Lippert, Euerdorf, Germany) and were derived from several long-standing colonies. All the pigs used were related to one another to varying degrees up to first degree. MH susceptibility or wild type was determined by DNA analysis (GeneControl GmbH, Poing, Germany) prior to the investigation. Homozygous cysteine for arginine substitution at position 615 of RYR1 indicating MH susceptibility was proven in nine pigs while eight animals were homozygous negative for this mutation.

After insertion of an intravenous line into an ear vein, general anesthesia was induced by application of fentanyl $(0.01 \mathrm{mg} / \mathrm{kg})$ (Janssen, Neuss, Germany) and thiopental (10 mg/kg) (Nycomed, Konstanz, Germany). Afterwards, the trachea was intubated without administration of a muscle relaxant using an orotracheal tube $9.0 \mathrm{~mm}$ internal diameter (Teleflex Medical GmbH, Kernen, Germany). The pigs were mechanically ventilated with an inspiratory oxygen fraction of 0.3 . Ventilator settings were adjusted to keep an end-tidal $\mathrm{PCO}_{2}$ of $32-39 \mathrm{mmHg}$ (respiratory-rate: 10-16 breaths/min; tidal volume: $8-12 \mathrm{ml} / \mathrm{kg}$; positive end-expiratory pressure: $5 \mathrm{mmHg}$ ). Anesthesia was maintained by continuous intravenous administration of thiopental $(10 \mathrm{mg} / \mathrm{kg} / \mathrm{h})$ and fentanyl $(0.01-0.02 \mathrm{mg} / \mathrm{kg} / \mathrm{h})$. Radiant heat application and warming blankets were used to ensure normothermia. Vital parameters were monitored continuously by invasive blood pressure monitoring in the tibial artery and by peripheral oxygen saturation, electrocardiography, and rectal temperature measurements.

After induction of general anesthesia, muscle specimens were excised from the left distal biceps femoris muscle, placed in carboxygenated (95\% oxygen, 5\% carbon dioxide) Krebs-Ringer's solution ( $\mathrm{NaCl} 118.1 \mathrm{mM}$; $\mathrm{KCl} 3.4 \mathrm{mM} ; \mathrm{CaCl}_{2} 2.5 \mathrm{mM} ; \mathrm{MgSO}_{4} 0.8 \mathrm{mM} ; \mathrm{KH}_{2} \mathrm{PO}_{4}$ $1.2 \mathrm{mM} ; \mathrm{NaHCO}_{3} 25.0 \mathrm{mM}$; Glucose $11.1 \mathrm{mM}$ ) (Pharmacy of the University Hospital, Wuerzburg, Germany) and immediately transported to the laboratory. Following preparation of the excised muscle into single muscle strips, length and wet weight of each muscle bundle was measured. For in vitro contracture test (IVCT) investigations, single muscle strips were mounted vertically in the experimental bath perfused with carboxygenated Krebs-Ringer's solution at $37^{\circ} \mathrm{C}$, fixed to an isometric force transducer (Hugo Sachs, Type 809, March, Germany) and stimulated electrically with a supra-maximal square wave stimulus at $1 \mathrm{~ms}$ duration and a frequency of $0.2 \mathrm{~Hz}$ (Hugo-Sachs-Elektronik, Type 215/I, March, Germany). Resting tension and twitch of the muscle strips were recorded continuously by a digital recording system (HSE-ACAD Software, Hugo Sachs, March, Germany). Only muscle specimens with initial twitch response of at least $10 \mathrm{mN}$ were included in the investigation. Two different muscle bundles per animal were analyzed. After stable conditions were reached, muscle bundles were incubated successively with increasing concentrations $(0.065$, $0.125,0.5,1.0,10$ and $50 \mu \mathrm{g} / \mathrm{ml}$ ) of a commercially available levosimendan preparation (Orion Pharma $\mathrm{GmbH}$, Hamburg, Germany) at $3 \mathrm{~min}$ intervals. To ensure sufficient viability of the muscle strips, investigations were performed within $3 \mathrm{~h}$ after muscle harvest.

After completion of the experiments, the pigs were euthanized in deep anesthesia by thiopental bolus application $(0.1 \mathrm{~g} / \mathrm{kg})$.

\section{Statistics}

Data are presented as median and interquartile range. A quasi-randomization was given by the availability of the animals independently from the MH diagnosis. Statistical evaluation was performed using D'Agostino \& Pearson test for normal distribution and student's t-test (length and weight of muscle strips, pre-drug resting tension and twitch force, maximum contracture) or 2-way-ANOVA with post-hoc Sidak's test for multiple comparisons (changes of twitch force, developing contractures) for differences between MHS 
and MHN groups. $P<0.05$ was considered statistically significant.

\section{Results}

The investigated muscle specimens did not differ between the groups regarding length (MHS: 22 [20-24] vs. MHN: 24 [21-24] mm; $p=0.40$ ), weight (MHS: 920 [820-1040] vs. MHN: 920 [740-1070] mg; $p=0.26$ ), initial twitch force (MHS: 25.1 [12.9-32.3] vs. MHN: 27.1 [16.5-31.7] $\mathrm{mN} ; p=0.84$ ) and pre-drug resting tension (MHS: 10.4 [8.5-17.3] vs. MHN: 12.6 [7.7-20.6] mN; $p=0.64)$. One of the two investigated muscle bundles per animal had to be excluded from the study because they failed to reach the pre-assigned minimum twitch force of $10 \mathrm{mN}$ in three MHS pigs and one MHN pig.

Incrementally increasing doses of levosimendan led to significantly different muscular reactions in the two investigated groups. With cumulative levosimendan concentrations $\leq 10 \mu \mathrm{g} / \mathrm{ml}$ an initial decrease of twitch amplitude in both groups was observed (at levosimendan $0.0625 \mu \mathrm{g} / \mathrm{ml}:$ MHS: -2.1 [-3.0- -0.9$]$ vs. $\mathrm{MHN}:-0.9$ [-1.8-0.1] $\mathrm{mN} ; p=0.99$; at levosimendan $10 \mu \mathrm{g} / \mathrm{ml}$ : MHS: $-7.1[-10.7-4.2]$ vs. MHN: -5.0 [-6.0- -3.2] $\mathrm{mN} ; p=0.26$ ), while at $50 \mu \mathrm{g} / \mathrm{ml}$ twitch amplitude was significantly higher in MHN (-3.0 [-5.2-0.2] mN) compared to MHS $(-7.5[-10.8--4.5] \mathrm{mN})(p=0.0034)$ muscle bundles (Fig. 1). In addition, no significant difference in baseline resting tension was detected between both groups at levosimendan $\leq 10 \mu \mathrm{g} / \mathrm{ml}$ (at levosimendan $0.0625 \mu \mathrm{g} / \mathrm{ml}$ : MHS: $-0.2[-0.6-0.2]$ vs. MHN: $-0.4[-1.3-0.1] \mathrm{mN} ; p=0.99$; at levosimendan $10 \mu \mathrm{g} / \mathrm{ml}:$ MHS: 1.7 [0.8-3.3] vs. MHN: -0.5 [-0.8-0.2] $\mathrm{mN} ; p=0.23$ ). However, exposure to levosimendan $50 \mu \mathrm{g} / \mathrm{ml}$ induced a significant higher muscular contracture in MHS

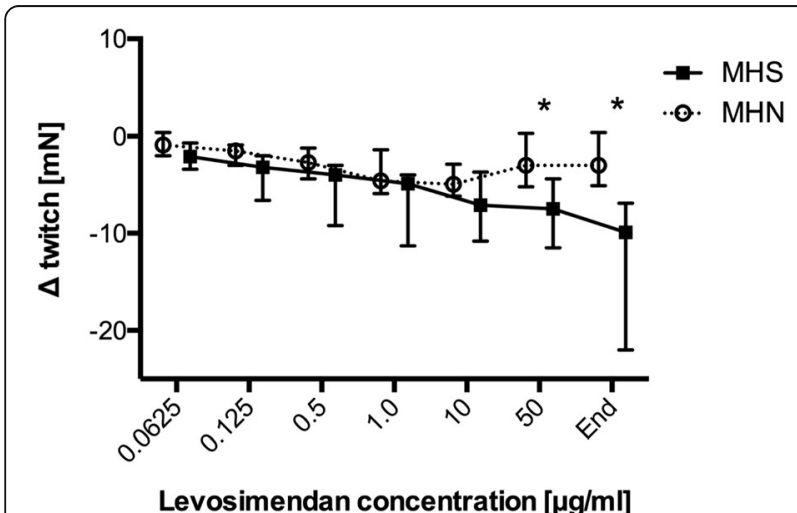

Fig. 1 Maximum twitch force after application of increasing levosimendan concentrations in MHS and MHN muscle.Data are presented as median and quartiles; $n=7$ for MHS; $n=12$ for MHN; $* P<0.05$ for differences between MHS and MHN. MHS: malignant hyperthermia susceptible; MHN: malignant hyperthermia non-susceptible compared to MHN muscle specimen (MHS: 3.3 [0.9-6.1] vs. MHN: $-0.7[-1.3-0.0] \mathrm{mN} ; p<0.0001$ ) (Fig. 2).

Analysis of maximum developing contractures over the whole range of applied levosimendan concentrations for every individual muscle bundle, regardless at which levosimendan concentration this maximum was achieved, showed significantly stronger muscular responses in MHS animals (MHS: (3.5 [2.3-7.6] vs. MHN: $1.2[0.5-1.8] \mathrm{mN} ; p=0.0006)$ (Fig. 3).

\section{Discussion}

Excessive sarcoplasmic calcium release is the underlying pathomechanism for $\mathrm{MH}$ crisis in susceptible individuals following exposure to trigger agents. This study investigated the influence of the calcium sensitizer levosimendan on skeletal muscle bundles of MHS and MHN swine in-vitro. Our results indicated that incubation with supra-therapeutic levosimendan doses leads to significant muscular contractures and decrease of twitch amplitude in MHS but increased twitch force in MHN muscle.

Based on $\mathrm{MH}$ associated mutations in the RYR1 or CACNA1S gene, triggering agents induce an excessive calcium efflux from the sarcoplasmic reticulum, resulting in a hypermetabolic state of the skeletal muscle cell, increased mitochondrial energy turnover and eventually metabolic failure. To date, various different $\mathrm{MH}$ causative mutations have been identified in humans, while in pigs $\mathrm{MH}$ phenotype is related to the homozygous mutation Arg615Cys in the RYR1 gene [8]. Thus, the porcine model is an established experimental set-up to investigate the pathophysiology of $\mathrm{MH}$.

IVCT is the gold standard for diagnosing $\mathrm{MH}$ susceptibility. In vitro incubation with calcium releasing drugs like volatile anesthetics or caffeine results in a pathological muscular contracture above a defined diagnostic

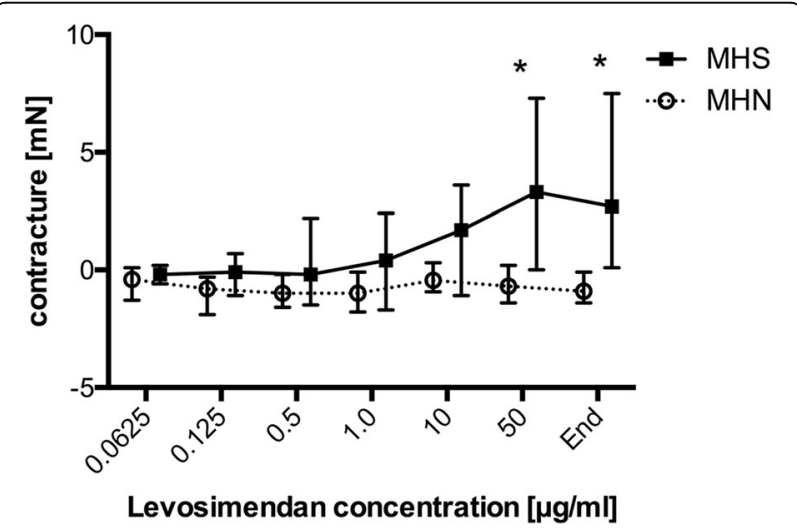

Fig. 2 Muscular contracture in comparison with baseline resting tension after levosimendan application in MHS and MHN. Data are presented as median and quartiles; $n=7$ for MHS; $n=12$ for $M H N ;{ }^{*} P<0.05$ for differences between MHS and MHN. MHS: malignant hyperthermia susceptible; MHN: malignant hyperthermia non-susceptible 


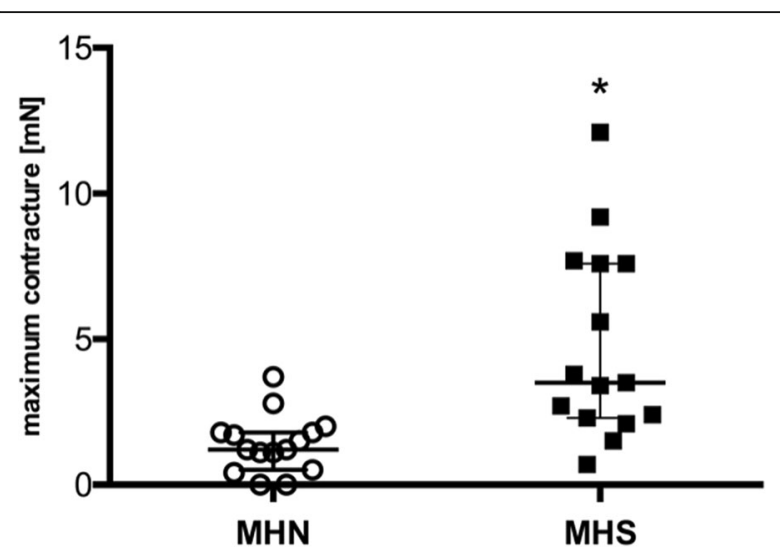

Fig. 3 Maximum contracture in MHS and MHN muscles over the whole range of applied levosimendan concentrations. Data are presented as median and quartiles; $n=7$ for $\mathrm{MHS} ; n=12$ for $\mathrm{MHN}$; ${ }^{*} P<0.05$ for differences between MHS and MHN. MHS: malignant hyperthermia susceptible; MHN: malignant hyperthermia non-susceptible

threshold in individuals susceptible to $\mathrm{MH}$. Moreover, IVCT serves as a solid model to analyze pathophysiological changes during an $\mathrm{MH}$ reaction in-vitro [9].

While $\mathrm{MH}$ triggering agents increase intracellular calcium concentrations by interaction with sarcoplasmic calcium release channels, levosimendan binds to slow skeletal and cardiac troponin $\mathrm{C}$ and stabilizes the conformation of the troponin complex [10]. Slow skeletal and cardiac troponin $C$ is also the dominant isoform in slow-twitch skeletal muscle fibers. Therefore, a levosimendan-induced enhancement of calcium sensitivity in slow-twitch skeletal muscle fibers seems reasonable. Levosimendan has been shown to induce a rise in submaximal force of slow-twitch diaphragm muscle fibers of patients with chronic obstructive pulmonary disease in-vitro and to improve diaphragm contractility of healthy volunteers in-vivo $[11,12]$. However, the proportion of slow-twitch muscle fibers in skeletal muscle varies among different muscle groups and individuals. For instance, in healthy humans, the soleus muscle contains more than $80 \%$ slow-twitch fibers, while in the quadriceps muscle only $50 \%$ are slow-twitch. In contrast, the proportion of slow-twitch fibers in vigorous adult pigs varies between $12 \%$ in the vastus lateralis muscle and up to $80 \%$ in postural muscles like the vastus intermedius muscle. In the biceps femoris muscle fiber distribution is rather homogeneous with a proportion of slow-twitch fibers of approximately $60 \%$ [13]. Therefore, biceps femoris muscle was selected for this investigation, assuming that this mainly slow-twitch muscle is particularly suitable for analyzing a possibly impact of levosimendan on the muscular response in MHS and $\mathrm{MHN}$ pigs.

Assessment of pharmacokinetics of intravenous levosimendan after a single bolus application of $2 \mathrm{mg}$ levosimendan revealed a plasma concentration of $0.3 \mu \mathrm{g} / \mathrm{ml}$ in healthy male volunteers [14]. To achieve sufficient tissue levels to provoke a reaction, in our study muscle bundles of MHS and MHN pigs were incubated with incremental concentrations of levosimendan starting from $0.0625 \mu \mathrm{g} / \mathrm{ml}$ up to $50 \mu \mathrm{g} / \mathrm{ml}$. A $3 \mathrm{~min}$ interval of exposure to each investigated levosimendan concentration was predefined, similar to the protocol for IVCT issued by the European $\mathrm{MH}$ Group [9].

To our surprise, muscle bundles of MHS pigs developed significantly stronger contractures at levosimendan levels of $50 \mu \mathrm{g} / \mathrm{ml}$ while twitch amplitude decreased significantly compared to MHN. This pathological contracture might be explained by a moderate increase of intracellular calcium levels caused by phosphodiesterase (PDE) inhibition as previously observed after exposure to supra-therapeutic levosimendan concentrations. The effects of levosimendan as selective PDE-III inhibitor outweighed the calcium sensitizing effects at higher concentrations [15]. However, these effects seem irrelevant within the limits of recommended therapeutic plasma levels [16]. Based on these findings our observations are in line with previous studies indicating that pharmacological inhibition of PDE-III by enoximone leads to contracture development in muscle bundles of MHS but not of MHN pigs in-vitro [17].

Maximum individual contractures were significantly higher in MHS compared to MHN muscle. Both, contracture development and decrease of twitch force may reflect a progressive intracellular metabolic breakdown in MHS muscles, which might be further amplified by preexisting higher resting calcium levels in the skeletal muscle of MHS pigs [18]. In contrast, MHN muscles showed a significant rise of the twitch amplitude suggesting an increase in muscular force caused by levosimendan. However, the observed effects were only detected at concentrations, which exceeded the therapeutic plasma levels by at least 150 times.

Interestingly, in MHS pigs conformational changes of cardiac troponin $\mathrm{C}$ and its integration into cardiac myofibrils were reported after levosimendan, which might affect the calcium dependency of myocardial contractility in the course of an $\mathrm{MH}$ episode [19]. Whether similar reactions in skeletal muscle of MHS individuals affect intracellular calcium homeostasis and lead to muscular contractions remains unclear so far.

\section{Conclusions}

In conclusion, the results of our investigation demonstrate that levosimendan dose-dependently causes significant contractures in MHS but not in MHN muscle in-vitro. Necessary levosimendan concentrations to induce this effect highly exceeded therapeutic plasma levels. Based on these observations, it seems unlikely that levosimendan at clinically relevant doses provokes $\mathrm{MH}$ crisis and poses a risk to susceptible individuals. 


\section{Abbreviations}

ANOVA: Analysis of variance; CACNA1S: Dihydrophyridine receptor; IVCT: In vitro contracture test; $\mathrm{MH}$ : Malignant hyperthermia; MHN: Malignant hyperthermia-non-susceptible; MHS: Malignant hyperthermia-susceptible; RYR1: Ryanodine receptor subtype 1

\section{Acknowledgements}

Chief technician Judith Skirde (Malignant Hyperthermia laboratory, University of Wuerzburg, Germany) contributed essential advice and technical assistance throughout the study.

The study was performed at the Department of Anesthesia and Critical Care at the University of Wuerzburg, Germany.

The results of this investigation were presented in part at the Annual Meeting of the European Malignant Hyperthermia Group in Lille, France, June 2015.

\section{Funding}

Publication of this investigation was funded by the German Research Foundation (DFG) and the University of Wuerzburg in the funding programme Open Access Publishing. The funding bodies had no role in the design of the study and collection, analysis, and interpretation of data and in writing the manuscript.

\section{Availability of data and materials}

The datasets used and/or analysed during the current study are available from the corresponding author on reasonable request.

\section{Authors' contributions}

FS conceived the study, accompanied the data acquisition, collected and analyzed the data and drafted the manuscript. SJ participated in the design of the study, performed the experiments, collected and analyzed the data and helped writing the manuscript. SI performed the experiments, collected and analyzed the data. IT performed the experiments, collected and analyzed the data. NR participated in the design of the study. All authors read and approved the final manuscript.

\section{Ethics approval}

The study was approved by the local animal care committee (Government of Unterfranken, Wuerzburg, Germany, No. 39/13)

\section{Consent for publication}

Not applicable.

\section{Competing interests}

The authors declare that they have no competing interests.

\section{Publisher's Note}

Springer Nature remains neutral with regard to jurisdictional claims in published maps and institutional affiliations.

Received: 26 June 2018 Accepted: 22 November 2018

Published online: 03 December 2018

\section{References}

1. Follath F, Cleland JG. Just H, Papp JG, Scholz H, Peuhkurinen K, Harjola VP, Mitrovic V, Abdalla M, Sandell EP, Lehtonen L; steering committee and investigators of the Levosimendan infusion versus Dobutamine (LIDO) study. Efficacy and safety of intravenous levosimendan compared with dobutamine in severe low-output heart failure (the LIDO study): a randomised double-blind trial. Lancet. 2002;360:196-202.

2. Edes I, Kiss E, Kitada Y, Powers FM, Papp JG, Kranias EG, Solaro RJ. Effects of Levosimendan, a cardiotonic agent targeted to troponin C, on cardiac function and on phosphorylation and $\mathrm{Ca} 2+$ sensitivity of cardiac myofibrils and sarcoplasmic reticulum in Guinea pig heart. Circ Res. 1995;77:107-13.

3. Papp Z, Édes I, Fruhwald S, De Hert SG, Salmenperä M, Leppikangas H, Mebazaa A, Landoni G, Grossini E, Caimmi P, Morelli A, Guarracino F, Schwinger RH, Meyer S, Algotsson L, Wikström BG, Jörgensen K, Filippatos G, Parissis JT, González MJ, Parkhomenko A Yilmaz MB, Kivikko M, Pollesello P, Follath F. Levosimendan: molecular mechanisms and clinical implications: consensus of experts on the mechanisms of action of levosimendan. Int J Cardiol. 2012;159:82-7
4. van Hees HW, Andrade Acuña G, Linkels M, Dekhuijzen PN, Heunks LM. Levosimendan improves calcium sensitivity of diaphragm muscle fibres from a rat model of heart failure. Br J Pharmacol. 2011;162:566-73.

5. Bruells CS, Marx G, Rossaint R. Ventilator-induced diaphragm dysfunction: clinically relevant problem. Anaesthesist. 2014;63:47-53.

6. Ochala J, Li M, Ohlsson M, Oldfors A, Larsson L. Defective regulation of contractile function in muscle fibres carrying an E41K beta-tropomyosin mutation. J Physiol. 2008;586:2993-3004.

7. Litman RS, Griggs SM, Dowling JJ, Riazi S. Malignant hyperthermia susceptibility and related diseases. Anesthesiology. 2018;128:159-67.

8. Rosenberg H, Pollock N, Schiemann A, Bulger T, Stowell K. Malignant hyperthermia: a review. Orphanet J Rare Dis. 2015;10:93.

9. Hopkins PM, Rüffert H, Snoeck MM, Girard T, Glahn KP, Ellis FR, Müller CR, Urwyler A. European malignant hyperthermia group. European malignant hyperthermia group guidelines for investigation of malignant hyperthermia susceptibility. Br J Anaesth. 2015;115:531-9.

10. Sorsa T, Pollesello P, Permi P, Drakenberg T, Kilpeläinen I. Interaction of levosimendan with cardiac troponin $C$ in the presence of cardiac troponin I peptides. J Mol Cell Cardiol. 2003;35:1055-61.

11. van Hees HW, Dekhuijzen PN, Heunks LM. Levosimendan enhances force generation of diaphragm muscle from patients with chronic obstructive pulmonary disease. Am J Respir Crit Care Med. 2009:179:41-7.

12. Doorduin J, Sinderby CA, Beck J, Stegeman DF, van Hees HW, van der Hoeven JG, Heunks LM. The calcium sensitizer levosimendan improves human diaphragm function. Am J Respir Crit Care Med. 2012;185:90-5.

13. Wank V, Fischer MS, Walter B, Bauer R. Muscle growth and fiber type composition in hind limb muscles during postnatal development in pigs. Cells Tissues Organs. 2006;182:171-81.

14. Chu KM, Hu YP, Liou JT. A pharmacokinetic and Pharmacodynamic study of intravenous Levosimendan in healthy Chinese volunteers and ethnic comparisons. Acta Cardiol Sin. 2014:30:298-307.

15. Pathak A, Lebrin M, Vaccaro A, Senard JM, Despas F. Pharmacology of levosimendan: inotropic, vasodilatory and cardioprotective effects. J Clin Pharm Ther. 2013;38:341-9.

16. Szilágyi S, Pollesello P, Levijoki J, Kaheinen P, Haikala H, Edes I, Papp Z. The effects of levosimendan and OR-1896 on isolated hearts, myocyte-sized preparations and phosphodiesterase enzymes of the Guinea pig. Eur J Pharmacol. 2004;486:67-74

17. Fiege M, Wappler F, Weisshorn R, Gerbershagen MU, Kolodzie K, Schulte am Esch J. Phosphodiesterase-III-inhibition with amrinone leads to contracture development in skeletal muscle preparations of malignant hyperthermia susceptible swine. Eur J Anaesthesiol. 2005;22:283-8.

18. Altamirano F, Eltit JM, Robin G, Linares N, Ding X, Pessah IN, Allen PD, López JR. Ca2+ influx via the $\mathrm{Na}+/ \mathrm{Ca} 2+$ exchanger is enhanced in malignant hyperthermia skeletal muscle. J Biol Chem. 2014;289:19180-90.

19. Liou YM, Jiang MJ, Wu MC. Changes in thiol reactivity and extractability of myofibril bound cardiac troponin C in porcine malignant hyperthermia. J Biochem. 2002:132:317-25.
Ready to submit your research? Choose BMC and benefit from:

- fast, convenient online submission

- thorough peer review by experienced researchers in your field

- rapid publication on acceptance

- support for research data, including large and complex data types

- gold Open Access which fosters wider collaboration and increased citations

- maximum visibility for your research: over $100 \mathrm{M}$ website views per year

At BMC, research is always in progress.

Learn more biomedcentral.com/submissions 